\title{
Isolation and Characterization of Mucoid and Non-Mucoid Salmonella oranienburg Isolated from Pleural Effusion of a Patient with Hemolytic Anemia
}

\author{
Yoshiniko Saitoh, Tatsuya Sasaki, Mieko Hiratsuka, \\ Nobuko Sato and Nobuhisa Yamane \\ Clinical Microbiology Branch, Central Clinical Laboratory, \\ Tohoku University School of Medicine, Sendai 980
}

\begin{abstract}
Saitoh, Y., Sasaki, T., Hiratsuka, M., Sato, N. and Yamane, N. Isolation and Characterization of Mucoid and Non-Mucoid Salmonella oranienburg Isolated from Pleural Effusion of a Patient with Hemolytic Anemia. Tohoku J. exp. Med., 1982, 136 (4), 379-386 — Salmonella oranienburg, mucoid and non-mucoid strains, were simultaneously isolated as causative agents from the pleural effusion of a patient with hereditary spherocytosis. The mucoid-typed Salmonella oranienburg was characterized by serological examination, taking advantage of the high frequency of mucoid (M) to non-mucoid (N) variation. Salmonella oranienburg; M-N variation; pleurisy; hemolytic anemia
\end{abstract}

Salmonellosis, including typhoid fever, has been regarded as an opportunistic infection, and has shown various clinical manifestations (Eisenberg et al. 1955; Hook 1961; Schneirson et al. 1964). Recently, we isolated Salmonella (S.) oranienburg from the pleural effusion of a patient with a 10 year history of hereditary spherocytosis. Up to this date, there have been many reports that salmonellosis was frequently complicated by hemolytic anemia, such as glucose-6-phosphate dehydrogenase deficiency (Lampe et al. 1975; Luzzatto 1975; Holland and Gelfand 1977; Kujwalile et al. 1979), sickle cell anemia (Chaturvedi et al. 1978; Adeyokunnu and Hendrickse 1980) and malaria (Schmitz and Gelfand 1976). Our observation in this communication also indicated a characteristic relationship between hemolytic anemia and Salmonellosis. In addition, mucoid and non-mucoid strains of $S$. oranienburg were simultaneously isolated. In general, it was impossible to identify the antigens $\mathrm{O}, \mathrm{Vi}$ and $\mathrm{H}$ of mucoid Salmonella, because there was no reaction occurred to the specific antisera. However, we were successful in the characterization of our isolate, taking advantage of the high frequency of mucoid to non-mucoid variation on agar and in semisolid agar.

\section{Case Report}

A forty-seven-year-old male was admitted to the hospital because of chest pain, fever, chills, fatigue, and dyspnea of three weeks duration. One month prior to 
admission he began to run a fever and to complain of a productive cough. He had been treated for hereditary spherocytosis for 10 years, and hepato-splenomegaly had been diagnosed when he was 20 years old. At admission, he appeared chronically ill and dyspneic. The heart rate was 126 beats/min, respiration 30 per min, blood pressure $96 / 50 \mathrm{~mm}$ of $\mathrm{Hg}$, and temperature $37.5^{\circ} \mathrm{C}$. A chest X-ray indicated the presence of a great volume of pleural effusion in the left-side. $\mathrm{He}$ underwent pleural puncture, and $1,500 \mathrm{ml}$ of fluid was collected. The pleural fluid taken contained many neutrophils and $3.4 \mathrm{~g} / \mathrm{ml}$ of protein, and showed positive Rivalta reaction. A culture of the pleural effusion revealed a Gram-negative rod, later identified as $S$. oranienburg.

The patient was initially treated with sulbenicillin (SB-PC) and cephalothin (CET) systemically, and gentamicin (GM) and continuous pleural drainage locally. Then, cefazolin (CEZ) and gamma-globulin were added. After one month of treatment, the pleural effusion disappeared. During his hospitalization, the blood analysis revealed marked anemia $(8.0 \mathrm{~g} / \mathrm{ml}$ of hemoglobin), reticulocytosis $(100 \%)$ and spherocytosis. He was discharged after 5 months.

\section{Bacteriological Observation}

Bacteriological examination of pleural effusion. The pleural fluid was purulent, greenish-yellow and very sticky, but did not contain any fresh blood. The microscopic examination revealed many neutrophils and Gram-negative rods. After inoculation on rabbit blood agar and Drigalski BTB lactose (BTB) agar and incubation at $37^{\circ} \mathrm{C}$ for overnight, there appeared two different colonies on both agars (Fig. 1). One was mucoid, which was the majority of the primary isolate,

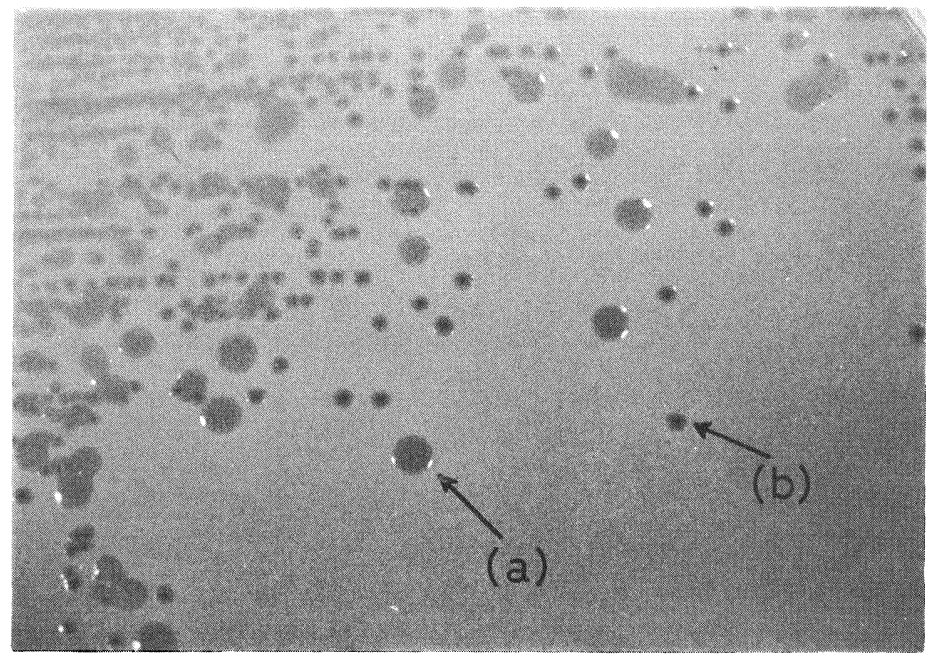

Fig. 1. Morphological features of mucoid and non-mucoid colonies of Salmonella oranienburg on BTB agar.

(a): Mucoid colony. (b): Non-mucoid colony. 
and the other was a normal, non-mucoid colony. Both isolates were Gramnegative rods.

In the standard biochemical reactions, both Gram-negative rods were identical, however the mucoid isolate lacked motility in sulfide-indole-motility (SIM) medium. The results indicated the characteristics of the Salmonella species, which is non-lactose-fermenting, produces $\mathrm{H}_{2} \mathrm{~S}$ in the deep of triple sugar iron (TSI) agar, utilizes citrate as a carbon source, and does not produce urease (see Table 1). Subsequently, the serological grouping with the specific antisera to the reference $\mathrm{O}, \mathrm{Vi}$ and $\mathrm{H}$ antigens, donated by Toshiba Kagaku Institute Company, Ltd., was performed. The non-mucoid colony was easily shown to have an antigenic configuration of $\mathrm{C}_{1}$ for $\mathrm{O},(-)$ for $\mathrm{Vi}$ and $\mathrm{m}$ and $\mathrm{t}$ for $\mathrm{H}$. The results indicated that the non-mucoid isolate was S. oranienburg (Wilson and Miles 1975). However, as is well known, the mucoid isolate did not show any agglutination to the antisera tested.

TABLE 1. Biochemical identification of mucoid and non-mucoid colonies of Salmonella oranienburg

\begin{tabular}{l} 
Biochemical reaction \\
\hline Catalase \\
Oxidase \\
TSI agar \\
H$_{2} \mathrm{~S}$ (TSI agar) \\
Indole \\
Citrate (Simmons) \\
VP \\
MR \\
IPA \\
Urease (Christensen) \\
Malonate \\
Lysine decarboxylase (LIA) \\
Tartrate (Jordan) \\
Motility
\end{tabular}

$\begin{array}{cc}\text { Mucoid form } & \text { Non-mucoid form } \\ + & + \\ - & - \\ \text { alkali/acid-gas } & \text { alkali/acid-gas } \\ + & + \\ - & - \\ + \text { (weak }) & + \\ - & - \\ + & + \\ - & - \\ - & - \\ - & - \\ + & + \\ + & + \\ - & +\end{array}$

TSI, triple sugar iron agar; VP, Voges-Proskauer reaction; MR, methyl red test; IPA, indole, pyruvic acid reaction; LIA, lysine iron agar.

Further characterization of mucoid Salmonella species. During several passages of the mucoid isolate, it was observed that, after the inoculation of the wellisolated mucoid colony to the agar, there appeared non-mucoid colonies in addition to the great majority of mucoid colonies. In order to characterize this new type of colony, we employed two different procedures to isolate the non-mucoid colony in pure form from the mucoid isolate (Fig. 2).

(1) The well-isolated mucoid colony was suspended in sterilized phosphate buffer saline ( $\mathrm{pH} 7.4)$ at an adequate cell concentration. The cell suspension was spread quantitatively on heart infusion (HI) agar. After incubation at $37^{\circ} \mathrm{C}$ for $18 \mathrm{hr}$ non-mucoid colonies appeared without fail with a $3.0-5.0 \%$ ratio to the total colony count of the majority of the mucoid colonies. The well-isolated non-mucoid colonies were individually subcultured to the BTB agar three times to obtain pure 


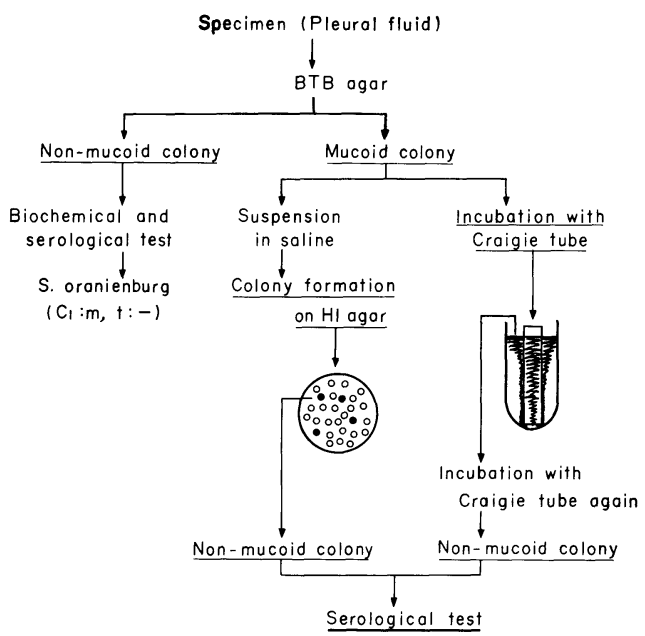

Fig. 2. The procedures to isolate the non-mucoid, high motility strains from the original mucoid colony.

culture isolates. The biochemical and serological testings revealed the same characteristics as the non-mucoid $S$. oranienburg originally isolated from the pleural effusion.

(2) The well-isolated mucoid colony was inoculated into semisolid cystinetrypticase agar (CTA) medium with Craigie's tube. This method has usually been employed to obtain high-motility subculture isolates. After inoculation into Craigie's tube and incubation at $37^{\circ} \mathrm{C}$ for $72 \mathrm{hr}$, some cloudy swelling reached the outer surface through the bottom of Craigie's tube. During three repetitions, the inoculate reached the outer surface within $20 \mathrm{hr}$, and the colonies looped from the surface were found to be non-mucoid on the BTB agar, and were reactive to antisera to $\mathrm{C}_{1}, \mathrm{t}$ and $\mathrm{m}$, the same as the former isolate.

Consequently, we characterized both mucoid and non-mucoid Salmonella species as S. oranienburg.

Susceptibility tests to some antibiotics. The antibiotic susceptibility tests were employed to mucoid, non-mucoid and non-mucoid derived from mucoid colony by two different methods. To antibiotics including chloramphenicol (CP), ampicillin (AB-PC) and sulfamethoxazole-trimethoprim (ST), there was no significant difference between these isolates in the agar diffusion susceptibility tests. The results revealed the high sensitivities to the antibiotics applied. However, when we employed the newly synthesized penicillin derivative, pivmecillinam (MPC), to the agar diffusion test, a great difference between mucoid and non-mucoid isolates was found (Fig. 3). The non-mucoid colony and the non-mucoid derived from mucoid colony were shown to be highly sensitive, but the mucoid colony appeared to be resistant. With the agar dilution method, we determined the minimum inhibitory concentration (MIC) to MPC. The non-mucoid colony showed less than $0.2 \mu \mathrm{g} / \mathrm{ml}$, but the mucoid colony $6.25 \mu \mathrm{g} / \mathrm{ml}$ as MIC value to MPC. The non-mucoid colonies 


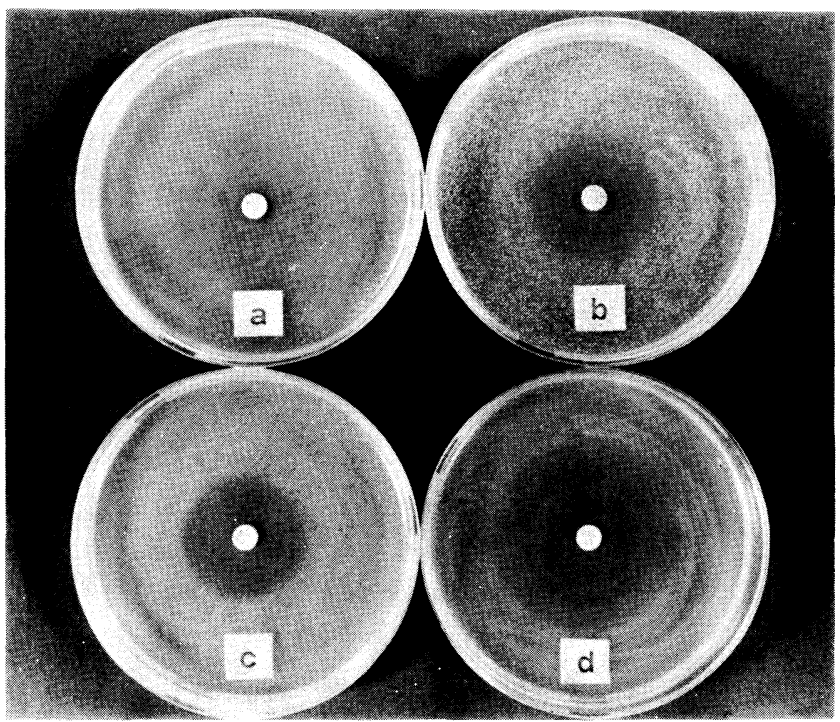

Fig. 3. Antibiotic susceptibility tests to pivmecillinam (MPC) by agar diffusion method.

(a): Mucoid strain originally isolated from the specimen.

(b): Non-mucoid strain originally isolated from the specimen.

(c): Non-mucoid strain derived from the mucoid colony on heart infusion (HI) agar (see text).

(d): Non-mucoid, high motility strain derived from the mucoid colony using Craigie's tube (see text).

derived from mucoid colony showed $1.56 \mu \mathrm{g} / \mathrm{ml}$.

\section{Discussion}

We have reported the isolation of $S$. oranienburg from the pleural effusion of a patient with hereditary spherocytosis. The case described has bacteriological and chemotherapeutic interest. First, mucoid and non-mucoid S. oranienburg were simultaneously isolated from the same specimen, and second, taking advantage of an unusually high frequency of mucoid to non-mucoid variation in vitro, it became possible to identify serologically the mucoid colony. Additionally, the susceptibility to certain antibiotic, i.e. pivmecillinam, differed by strains, corresponding to the mucoid to non-mucoid variation.

It has been well recognized that systemic, or local salmonellosis occurred with increased frequency in hemolytic anemia, including sickle cell anemia (Lampe et al. 1975; Luzzatto 1975; Holland and Gelfand 1977; Chaturvedi et al. 1978; Kujiwalile et al. 1979; Adeyokunnu and Hendrickse 1980) and malaria (Schmitz and Gelfand 1976), however, this predisposition to Salmonella infection is poorly understood. Hand and King $(1977,1978)$ reported the deficiency of the sickel cell anemia patient in the bacteriocidal function of sera and explained with the defective function of the alternative complement pathway, low serum C3 concentration, and decreased total iron-binding capacity. In our case, unfortunately a detailed examination was 
not performed. However, Salmonella, including S. oranienburg, appears to be a specific pathogen in hereditary spherocytosis.

Also, it was of considerable interest that the mucoid and non-mucoid forms were isolated simultaneously, and the form change from mucoid to non-mucoid was observed in vitro in a high ratio, although the reverse change was not. This facilitated the serological identification of the mucoid-form S. oranienburg. The spontaneous variation from the mucoid to the non-mucoid colony form is often observed in strains of Pseudomonas aeruginosa, but the reverse has been rarely reported (Iacocea et al. 1963; Burns and May 1968; Doggett 1969; Diaz and Neter 1970). The phenomenon observed in this report paralleled closely to the findings in Pseudomonas. However, the mucoid and non-mucoid transformation mechanism of this emergence is presently not understood. A number of reports suggested that the mucoid variation is probably not related to a plasmid DNA, and could be linked to a phage in strains of Pseudomonas aeruginosa (Martin 1973; Markowitz et al. 1978), Escherichia coli (Sapelli and Goebel 1964) and Brucella abortus (Jones et al. 1962). The evidence is accumulating that the change from non-mucoid to mucoid colonies may occur in the human body (Doggett et al., 1966; Daiz and Neter 1970), and the non-mucoid always preceded the mucoid in patients, suggesting that naturally occurring mucoid forms were selected in the presence of phage in the lytic cycle.

Mucoid strains have been shown to possess distinct polysaccharides which differ from the non-mucoid extra-cellular materials containing the antigenic determinants of $\mathrm{O}$ (Carlson and Matthews 1966; Bartell et al. 1970). This difference might be reflected in the susceptibility to some antibiotics. If this is the case, the possible structural analogies or a more complex biological mechanism, such as plasmids or membrane permeability, might serve as explanations for this difference. However, if we assume that, contrary to the results of susceptibility to other penicillin derivatives, only pivmecillinam sensitivity changed in correspondence to the mucoid to non-mucoid variation, the unique biological action of pivmecillinam to the mucoid colonies might be proposed. As a preliminary analysis, it was demonstrated that the bacteriocidal mechanism is quite different from that of other pencillins, especially in the bacteriolysis process (Nakazawa et al. 1977), Thus, it may be suggested that the difference in pivmecillinam sensitivity between the mucoid and non-mucoid strains is the reflection of the difference of the final process, bacteriolysis, probably in the combination of lysis with antibiotic and phage.

\section{Acknowledgments}

We would like to thank Miss M. Yuki for her technical assistance, and Toshiba Kagaku Institute Co., Ltd., for the donation of antisera. This study was supported in part by the Sendai Institute of Microbiology. 


\section{References}

1) Adeyokunnu, A.A. \& Hendrickse, R.G. (1980) Salmonella osteomyelitis in childhood. A report of 63 cases seen in Nigerian children of whom 57 had sickle cell anemia. Arch. Dis. Child., 55, 175-184.

2) Bartell, P.F., Orr, T.E. \& Chudio, B. (1970) Purification and chemical composition of the protective slime antigen of Pseudomonas aeruginosa. Infect. Immun., 2, 543-548.

3) Burns, M.W. \& May, J.R. (1968) Bacterial precipitins in serum of patients with cystic fibrosis. Lancet, 1, 270-272.

4) Carlson, D.M. \& Matthews, L.W. (1966) Polyuronic acids produced by Pseudomonas aeruginosa. Biochemistry, 5, 2817-2822.

5) Chaturvedi, P., Jain, V.K., Gupta, R.K., Saoji, S., Moghe, K.V. \& Sharma, S.M. (1978) Sickle cell anaemia with Salmonella empyema thoracis. Indian Pediatr., 15, 605-606.

6) Diaz, F. \& Neter, E. (1970) Pseudomonas aeruginosa: serogroups and antibody response in patients with neoplastic diseases. Amer. J. med. Sci., 259, 340-345.

7) Doggett, R.G. (1969) Incidence of mucoid Pseudomonas aeruginosa from clinical sources. Appl. Microbiol., 18, 936-937.

8) Doggett, R.G., Harrison, G.M., Stillwell, R.N. \& Wallis, E.S. (1966) An atypical Pseudomonas aeruginosa associated with cystic fibrosis of the pancreas. J. Pediat., 68, 215-221.

9) Eisenberg, G.M., Palazzola, A.J. \& Flippin, H.F. (1955) Clinical and microbiologic aspects of salmonellosis: study of ninety-five cases in adults and children. New Engl. J. Med., 253, 90-94.

10) Hand, W.L. \& King, N.L. (1977) Deficiency of serum bacteriocidal activity against Salmonella typhimurium in sickle cell anaemia. Clin. exp. Immunol., 30, 262-270.

11) Hand, W.L. \& King, N.L. (1978) Serum opsonization of salmonella in sickle cell anemia. Amer. J. Med., 64, 388-395.

12) Holland, S. \& Gelfand, M. (1977) The clinical associations of glucose-6-phosphate dehydrogenase (G-6-PD) deficiency. A study at Harare Hospital. Cent. Afr. J. Med., 23, 285-289.

13) Hook, E.W. (1961) Salmonellosis: certain factors influencing interaction of salmonella and human host. Bull. N.Y. Acad. Med., 37, 499-512.

14) Iacocca, V.F., Sibinga, M.S. \& Barbero, G.J. (1963) Respiratory tract bacteriology in cystic fibrosis. Amer. J. Dis. Child., 106, 315-324.

15) Jones, Lois M., McDuff, C.R. \& Wilson, J.B. (1962) Phenotypic alterations in the colonial morphology of Brucella abortus due to a bacteriophage carrier state. $J$. Bact., 83, 860-866.

16) Kujwalile, J.M., Kigoni, E.P. \& Nhonoli, A.M. (1979) Frequency of glucose-6phosphate dehydrogenase (G-6-PD) deficiency in Tanzanian males with typhoid fever. Trop. Geogr. Med., 31, 225-228.

17) Lampe, R.M., Kirdpon, S., Mansuwan, P. \& Benenson, M.W. (1975) Glucose-6phosphate dehydrogenase deficiency in Thai children with typhoid fever. $J$. Pediat., 87, 576-578.

18) Luzzatto, L. (1975) Inherited haemolytic states: glucose-6-phosphate dehydrogenase deficiency. Clin. Haematol., 4, 83--108.

19) Markowitz, S.M., Macrina, F.L. \& Phibbs, P.V. (1978) R-factor inheritance and plasmid content in mucoid $P$. aeruginosa. Infect. Immun., 22, 530-539.

20) Martin, D.R. (1973) Mucoid variation in P. aeruginosa induced by the action of phage. J. med. Microbiol., 6, 111-118.

21) Nakazawa, S., Nishino, T. \& Yamagishi, J. (1977) Morphological study on antimicrobial activities of Mecillinam against Escherichia coli. Chemotherapy, 25, 46-60 (in Japanese with English abstract).

22) Sapelli, R. V. \& Goebel, W.F. (1964) The capsular polysaccharide of a mucoid variant of E. coli K 12. Proc. nat. Acad. Sci. USA, 52, 265-271.

23) Schmitz, B. \& Gelfand, M. (1976) A study of the clinical features of malaria in 
Rhodesia. Part III. African children. Cent. Afr. J. Med., 22, 134-137.

24) Schneirson, S.S., Herschberger, C. \& Honigsbery, R. (1964) Salmonellosis at Mount Sinai Hospital: ten year survey (1953-1963). J. Mt. Sinai Hosp., 31, 1-9.

25) Wilson, G.S. \& Miles, A.A. (1975) Salmonella. In: Principles of Bacteriology, Virology and Immunity. 6th ed., Edward Arnold (Publishers), Ltd., London, pp. 918-955. 